Adherence to recommendation 2.6 .3 of the service specification for Tier 4 CAMHS was assessed. Standard 2.6.3 of the service specification for Tier 4 child and adolescent mental health services states that "on admission all young people must have an initial assessment (including a risk assessment) and care-plan completed within 24 hours. Where admission is for day/in-patient care this will include a physical examination." In line with this standard this audit will evaluate the use of physical examination, baseline blood tests and ECG carried out on young people.

Background. Mental health problems in children and young people are associated with both short- and long-term physical health problems. It is therefore important that they undergo full physical health assessment on admission to a Tier 4 inpatient unit.

Method. Electronic records were reviewed for all patients admitted within a 6 months period, between 1st August 2018 and 1st February 2019. Data were collected in March 2019 and entered directly into an excel spread sheet designed for data collection. A total of 23 patients were identified for inclusion in this audit.

Simple statistical analysis was carried out using excel.

Result. Over $80 \%$ of patients who did not refuse had a completed physical examination (85\%), blood results recorded (82\%) and ECG (84\%) within the first 24 hours of their admission. 100\% of patients who did not refuse had bloods and ECG checked at some time during their admission, with $90 \%$ having a physical examination.

For several patients (3 physical examination, 2 bloods, 3 ECG), no reason was documented as to why the procedure or examination did not take place. For 1 patient, blood tests were delayed due to having no blood tubes available.

Conclusion. Taken into account the result of this audit and bearing in mind the importance of physical examination as part of the admission process, it is important to try and support both regular Mill Lodge staff and on-call junior doctors to follow Standard 2.6.3's guidance around physical examination on admission to hospital. While good results were seen in many areas, the ward is not yet achieving the standard of $100 \%$. A re-audit will take place in twelve months' time to review recommendation and compliance.

\section{Appropriateness of referrals to Whiston hospital Mental Health Liaison Services}

Nick Strouther* and Divya Jain

The Brooker Centre, HENW

${ }^{\star}$ Corresponding author.

doi: 10.1192/bjo.2021.931

Aims. 1. The aim of this study was to assess the appropriateness of referrals to Whiston Mental Health Liaison Services (WMHLS) according to Royal College of Psychiatrists and local trust guidelines.

2. To assess whether the referrals were being reviewed in timely manner as per the trust's guidelines.

Method. Data collection was completed using a proforma to ensure uniform data collection. The proforma included information on patient demographics, previous mental health service involvement, other details like reasons and time of referral and their outcomes. Data sample comprised of 46 patients who had been referred to the WMHLS in the month of August 2019 were randomly selected.

Result. 44 of the 46 referrals analyzed were found to be appropriate. 40 patients were deemed to have appropriate documentation. The ratio of males to females was 20:26. 21 referrals were from the observation ward, 14 from A\&E, and 11 from medical wards. 40 patients were previously known to mental health services. The reasons for referral ranged from suicidal ideation/attempt (48\%), Drug related (12\%), Assessment (7\%) and more. There were various outcomes recorded. One of them was that 18 (28\%) referrals were assessed for Depression and for other mental health problems.

$78.6 \%$ of patients referred from A\&E, and $95.2 \%$ of patients in the observation ward, were not seen in the 1 hour window set out by the Trust's guidelines. $91.1 \%$ of patients referred from the wards were seen within the 24 hour target.

Conclusion. The vast majority of referrals were found to be appropriate $(44 / 46)$. It was found that the referral form used across the Trust, contained different levels of details and information on the patient depending on the source of referral. Using a standard process to complete referral forms to be used across the whole trust may ensure that all patients receive a standardized and appropriate referral based on the guidelines. Making the form electronic may reduce problems deciphering handwriting, and could allow WMHLS have a better understanding of the patient, and allow them to identify a patient that may be more appropriate for another service, e.g. drugs and alcohol team. This may and make the overall referral process quicker and reduce waiting times in $\mathrm{A} \& \mathrm{E}$, as well as faster referrals to the appropriate services.

Audit of delays in the diversion of mentally disordered defendants under the Mental Health Act 1983/2007 at a liaison and diversion service in North West London

Seema Sukhwal ${ }^{1 \star}$, Claire Gordon-Ellis ${ }^{2}$ and Luneta Tajblova ${ }^{3}$

${ }^{1}$ Barnet Enfield and Haringey MH NHS Trust; ${ }^{2}$ Central and North West London NHS Foundation Trust and ${ }^{3}$ Together for Mental Wellbeing

${ }^{\star}$ Corresponding author.

doi: $10.1192 /$ bjo.2021.932

Aims. To ascertain the length of time defendants wait for a Mental Health Act assessment (MHAA) and where necessary, how long they are waiting for a hospital bed.

Background. The Liaison and Diversion Service in North West London (the Service) is provided by Central North West London Foundation NHS Trust (CNWL), Barnet Enfield Haringey (BEH) and Together to Willesden Magistrates Court in North West London.

One of the core activities of the Service is diverting individuals from the criminal justice system to hospital under the Mental Health Act (MHA).

The Code of Practice allows for a period of 14 days between the medical recommendation and conveyance to hospital. Defendants needing admission under MHA are remanded to custody if a bed is not available. This prevents them from receiving the assessment and care they need. We consider that all defendants found to be liable to detention under the MHA should be admitted to a hospital bed on the same day.

Method. Data were collected between October 2018 and February 2019. All patients referred for a MHAA were included. The time a MHAA was requested, took place as well as how long the defendant waited for a bed was noted.

Result. A total of $42 \mathrm{MHAA}$ were requested. 25 individuals were detained under Section 2 of the MHA 1983.

The time between referral for a MHAA and the MHAA taking place was obtained in 25 of the 42 referrals. The range of times 
between a referral being made and the assessment taking placed varied between 1.5 hours and 22 hours. Two defendants were remanded overnight in prison as the MHAA could not take place on the same day as the referral.

In the 25 cases where an application for detention under Section 2 of the MHA was made, beds were not available on the same day in 7 cases. In 4 cases defendants required remand in prison custody due to beds not being available.

Conclusion. There were some limitations to this audit as data were not available for all 42 individuals referred for a MHAA.

Individuals referred for MHAA by the Service had both medical recommendations completed within 5 days and those who required admission to hospital were admitted within 14 days of the recommendations being completed.

Whilst these standards are being met, individuals referred for MHAA and those requiring admission to hospital are still facing remand to custody.

\section{Handcuff use on forensic psychiatry wards}

Rachel Swain*, Deborah Klayman, David Reiss, Kruti Buch and Sarah Roberts

West London NHS Trust

${ }^{\star}$ Corresponding author.

doi: 10.1192/bjo.2021.934

Aims. This project aimed to assess the use of handcuffs in a secure forensic mental health hospital.

Background. Handcuffs are used by secure forensic psychiatric wards where patients need to leave the ward and require added restrictive measures for their own or other's safety. The decision to use handcuffs is made by the multi-disciplinary team, with the input of the unit's clinical security team and is assessed based on individual risk and need. This study investigated the frequency, duration and purpose of handcuff use in one secure forensic mental health unit, encompassing 8 male medium secure wards, 5 male low secure wards, 1 adolescent secure ward, 1 female low secure ward and 5 female medium secure wards.

Method. Handcuff use was recorded contemporaneously by ward staff in a specialised handcuff proforma. This data were then compiled to assess the number of instances of use, the mental health section applicable to the patient, the reason the patient needed to leave the unit, and the duration of use (including the time period for which the handcuffs were removed during the visit, if applicable.) Data from these forms over an 18 month period were analysed.

Result. Over the 18 months, there were a total of 347 uses of handcuffs, with an average of 18.3 occurrences per month. In 55 cases, the patients were detained under a civil section, with the remaining instances occurring in patients detained under forensic section. $47 \%$ were unsentenced prisoners.

The most common destination for patients was the general medical hospital, which accounted for $49 \%$ of all visits. Court was the second most common destination, with $39 \%$ of uses.

The average duration spent in handcuffs was 3.3 hours. The average time that the handcuffs were taken off during the transfer was 1.2 hours.

Conclusion. Through ongoing education and supervision by the clinical security team, handcuff use in this forensic service was limited to essential situations, most often to allow treatment of physical health issues off-site. A large proportion of instances involved unsentenced prisoners and court attendances, where the risk of absconsion might be particularly high. Duration spent in handcuffs was kept to a minimum, with cuffs being removed where possible. The service strives to continue such good practices and to identify further ways to reduce handcuff use, such as using videoconferencing as an alternative court attendance.

\section{Assessment and management of patients detained under Section 136 in Northwick Park Hospital emergency department}

\author{
Laurence Telesia ${ }^{1 \star}$ and Lauren Fraser ${ }^{2}$ \\ ${ }^{1}$ South London and Maudsley NHS Foundation Trust and \\ ${ }^{2}$ Northwick Park Hospital, London North West University \\ Healthcare NHS Trust \\ ${ }^{\star}$ Corresponding author.
}

doi: 10.1192/bjo.2021.935

Aims. To evaluate the role of the Emergency Medicine team (EM) within a London Emergency Department (ED) in assessing and managing patients detained under Section 136 of the Mental Health Act, 1983 (S136).

Background. S136 allows detention and transfer of people to ED and psychiatric hospitals for further assessment. EDs are optimised for the investigation and management of the medically unwell, but attending ED may also delay access to psychiatric services if required. Minimal research has been performed to investigate the relative benefits of transferring people under S136 to ED versus psychiatric hospitals.

Method. Electronic notes were searched to identify those attending under S136 between 01/04/2017 and 31/03/2018. Scanned medical notes were reviewed and data extracted regarding patient demographics, length of ED stay, reason for S136 use, investigations and interventions undertaken by EM.

Result. This identified 95 attendances by 87 patients. The mean age was 35 years (range 15-75) and 59\% of attenders were male. The mean duration of stay was 7 hours 34 minutes (range 6 minutes - 25 hours 50 minutes).

Reasons for S136 use were abnormal behaviour (32), expressed suicidal ideation (29), overdose (15), self-harm (13), overdose plus self-harm (4), being found wandering (1) and was unclear for 1 presentation.

In 39 attendances no investigations beyond history and examination were performed by EM. Only 6 patients had investigations that were not bloods, electrocardiogram or urinalysis. These included X-radiograph trunk (4), computed tomography (CT) head (3), X-radiograph limb (3), CT cervical spine (1), Focused Assessment with Sonography for Trauma (1).

No interventions were given by EM in 55 attendances. Twenty-nine different medications were prescribed and 18 patients were prescribed intravenous fluids. Three had wounds dressed, 3 glued, 3 sutured and 1 stapled.

Conclusion. There were difficulties categorising the reason for S136 use, as clear documentation was often unavailable, but the vast majority of patients were detained due to abnormal behaviour, expressed suicidal ideation and self-harm.

Few attending ED under S136 received investigations or interventions that could not be offered within a psychiatric hospital. There was a wide range in duration of stay within ED, however $65 \%$ of attendances were longer than the standard 4 hour target.

Future research may assess the relative benefits of ED versus psychiatric hospitals in assessing those detained. This could aid services in meeting both the physical and psychiatric needs of patients whilst making efficient use of available resources. 